\title{
Gene Expression Correlation for Cancer Diagnosis: A Pilot Study
}

\author{
Binbing Ling, ${ }^{1}$ Lifeng Chen, ${ }^{1}$ Qiang Liu, ${ }^{2}$ and Jian Yang' \\ ${ }^{1}$ Drug Discovery and Development Research Group, College of Pharmacy and Nutrition, University of Saskatchewan, \\ 107 Wiggins Road, Saskatoon, SK, Canada S7N 5E5 \\ ${ }^{2}$ Vaccine and Infectious Disease Organization-International Vaccine Centre, University of Saskatchewan, 120 Veterinary Road, \\ Saskatoon, SK, Canada S7N 5E3 \\ Correspondence should be addressed to Jian Yang; jian.yang@usask.ca
}

Received 6 December 2013; Accepted 11 March 2014; Published 9 April 2014

Academic Editor: FangXiang Wu

Copyright (c) 2014 Binbing Ling et al. This is an open access article distributed under the Creative Commons Attribution License, which permits unrestricted use, distribution, and reproduction in any medium, provided the original work is properly cited.

Poor prognosis for late-stage, high-grade, and recurrent cancers has been motivating cancer researchers to search for more efficient biomarkers to identify the onset of cancer. Recent advances in constructing and dynamically analyzing biomolecular networks for different types of cancer have provided a promising novel strategy to detect tumorigenesis and metastasis. The observation of different biomolecular networks associated with normal and cancerous states led us to hypothesize that correlations for gene expressions could serve as valid indicators of early cancer development. In this pilot study, we tested our hypothesis by examining whether the mRNA expressions of three randomly selected cancer-related genes PIK3C3, PIM3, and PTEN were correlated during cancer progression and the correlation coefficients could be used for cancer diagnosis. Strong correlations $(0.68 \leq r \leq 1.0)$ were observed between PIK3C3 and PIM3 in breast cancer, between PIK3C3 and PTEN in breast and ovary cancers, and between PIM3 and PTEN in breast, kidney, liver, and thyroid cancers during disease progression, implicating that the correlations for cancer network gene expressions could serve as a supplement to current clinical biomarkers, such as cancer antigens, for early cancer diagnosis.

\section{Introduction}

Cancer is a malignant neoplasm that kills about 75,500 Canadians in 2013 [1]. Taking advantage of recent progress in cancer treatments, especially in chemotherapy and targeted therapy, the age-standardized five-year relative survival rate (RSR) for Canadian cancer patients has increased from 56\% in the 1992-1994 period to 63\% in the 2006-2008 period [1]. However, prognosis for high-grade, late-stage, and recurrent cancers remains poor; for example, the five-year RSR for breast cancer dropped dramatically from $96 \%$ for stage I to $26 \%$ for stage IV [2]. Therefore, early diagnosis is still essential for cancer patient survival.

Cancer is characterized by uncontrolled cell growth as a consequence of activating protooncogenes and/or inactivating tumor suppressor genes [3-5]. Searching for consistently up- or downregulated genes, proteins, or clusters of genes has been the mainstream in identifying potential biomarkers for early cancer diagnosis [6-8]. Nevertheless, this approach experiences a key limitation that expression of cancer-related genes and proteins may change dramatically during disease progression, which could lead to misdiagnosis. Cancer cells are living in a hostile environment and undertaking constant attacks by the human immune system $[9,10]$. They need to network and coordinate many genes to counteract the host attacks for their own survival, and one of such networking examples is the crosstalk between different signaling pathways to enhance cancer proliferation, migration, and drug resistance [11-15]. With recent advances in high-throughput genomic and proteomic studies and significantly increased computing power, constructing and dynamically analyzing biomolecular networks to detect tumorigenesis, predict cancer progression, and even possibly guide cancer treatment has attracted a significant amount of research interest in recent years [16-19].

Tumorigenesis as well as cancer progression is a complex and dynamic process. A recent study on protein interaction networks showed that the biomolecular networks for cancer cells may be significantly different from those for 
normal cells [20]. This observation led us to hypothesize that increased correlations for the expressions of genes in the cancer networks associated with decreased correlations for the expressions of genes in the normal networks might serve as valid biomarkers for early diagnosis of tumorigenesis and cancer progression. In this pilot study, we randomly chose three cancer-related genes, PIK3C $3, P I M 3$, and PTEN, to test our hypothesis without knowing whether they are clustered together in any cancer network prior to a costly large scale patient-population based and cancernetwork confirmed clinical screening. PIK3C 3 encodes class III phosphatidylinositol 3-kinase (PIK3C3/Vps34), which works together with SH3GLB1/Bif-1, a haploinsufficient tumor suppressor, to form autophagosome to counteract oncogene-driven tumorigenesis [21, 22]. PIM3 is an oncogene encoding a $\mathrm{Ca}^{2+} /$ calmodulin-dependent protein kinase Pim3 , which inhibits cell apoptosis and promotes cell survival [23-25]. PTEN is a tumor suppressor gene that encodes PTEN (phosphatase and tensin homologue deleted from chromosome-10). PTEN catalyzes the dephosphorylation of PI $(3,4,5) \mathrm{P} 3$ (phosphatidylinositol 3,4,5-trisphosphate) to PI(3,4)P2 (phosphatidylinositol 3,4-bisphosphate), which, in turn, downregulates the AKT pathway to inhibit cell growth and induce cell apoptosis [26-28]. Although the three selected genes are neither clustered directly in any identified signaling pathway nor confirmed to be copresent in any cancer network, our limited but insightful analysis showed high correlations for the mRNA expressions among the three genes in breast cancer, between PIK3C 3 and PTEN in ovary cancer, and between PIM 3 and PTEN in kidney, liver, and thyroid cancers during disease progression.

\section{Materials and Methods}

The 96-sample TissueScan Oncology qPCR Cancer Survey Panel was purchased from the OriGene Technologies, Inc. (Rockville, MD, USA). Primer sequences for PIK3C3, PIM3, and PTEN (Table 1) were designed and synthesized by Applied Biosystems (Foster City, CA, USA) based on the ITS region. The TaqMan probe was labeled with FAM at $5^{\prime}$ end and with nonfluorescent quencher at $3^{\prime}$-end. The mRNA expression levels for genes PIK3C3, PIM3, and PTEN were evaluated with the 96-sample qPCR Cancer Survey Panel by quantitative real-time RT-PCR using an Applied Biosystems 7300 Real-Time PCR System. The expression for each gene was normalized to the internal control, $\beta$-actin, in different patients. The quantitative real-time RT-PCR reaction mixture consisted of TaqMan Gene Expression Master Mix (Applied Biosystems), $0.9 \mu \mathrm{M}$ of each primer for gene PIK3C3, PIM3, or PTEN and the ACTB gene encoding $\beta$-actin, and $0.9 \mu \mathrm{M}$ of the TaqMan probe. The PCR reaction mixture was then added to the 96-sample qPCR Cancer Survey Panel at $30 \mu \mathrm{L}$ per well. The amplification was carried out under the following conditions: $2 \mathrm{~min}$ at $50^{\circ} \mathrm{C}, 10 \mathrm{~min}$ at $95^{\circ} \mathrm{C}, 60$ cycles of $15 \mathrm{~s}$ at $95^{\circ} \mathrm{C}$, and finally $1 \mathrm{~min}$ at $60^{\circ} \mathrm{C}$. The expression level of each gene was averaged by disease stage and normalized to $\beta$-actin. The fold difference in mRNA expression at each disease stage was determined by comparison to expression levels in patients with noninvasive cancer (stage 0 , expression level set as 1). For each cancer type, unpaired $t$-test with Welch's correction between the averaged expression levels in noninvasive and invasive cancer patients was performed with $\alpha=0.05$ using GraphPad Prism 4.0 (GraphPad Software, San Diego, CA, USA).

\section{Results and Discussion}

Tumor development, progression, metastasis, and drug resistance require a delicately controlled gene network [29-32]. These genes are unlikely to play equally important roles in the network. Constructing and analyzing biomolecular networks using bioinformatic techniques has emerged to be a promising tool in identifying key players in the cancer networks. However, clinical validation of a cancer network is facing several challenges: (1) a large patient population taking into consideration the factors such as race, gender, age, and environment, nutrition, (2) high cost associated with the clinical screening, and (3) heterogeneity of the tumor tissues. Thus, a pilot study is always necessary and beneficial for such type of research.

Recently, Islam et al. reported that the biomolecular networks were significantly different between normal and cancerous states from their protein interaction studies [20]. Based on this report, we hypothesized that increased correlations for the expressions of a set of genes (cancer network) associated with decreased correlations for the expressions of another set of genes (normal network) might serve as an indicator for tumorigenesis and cancer progression. We undertook the current pilot study to test our hypothesis by evaluating the mRNA expressions of three randomly selected cancer-related genes, PIK3C3, PIM3, and PTEN, using quantitative real-time RT-PCR approach. The three selected genes are not directly clustered in any identified signal transduction pathway to our knowledge, and the reason for choosing quantitative real-time RT-PCR is that it is faster, more reliable, more quantifiable, and cheaper than protein analysis of patient samples. Although it is unknown whether the three genes are grouped together in any cancer network, observation of strong correlations among their mRNA expressions would definitely support our hypothesis that gene expression correlations were equally valid as the genes themselves to serve as biomarkers for diagnosis of tumorigenesis and cancer progression.

Ninety-six patient samples were included in this pilot study with twelve samples spreading over different disease stages for each of the eight types of cancer (see Supplementary Table S1 available online at http://dx.doi.org/10.1155/2014/ 253804). The mRNA expression levels of genes PIK3C3, PIM3, and PTEN were quantitatively analyzed by real-time RT-PCR. As shown in Table 2, the disease stage-averaged mRNA expressions (stages I to IV) for PIK3C3, PIM3, and $P T E N$ were elevated by 3.1 -fold $(P=0.01), 3.8$-fold $(P=$ $0.00)$, and 3.1-fold $(P=0.04)$, respectively, in breast cancer. The disease stage-averaged mRNA expression for PIK3C3 was downregulated by 2 -fold $(P=0.03)$ in thyroid cancer. No statistically significant up- or downregulation of the 
TABle 1: Primer and TaqMan probe sequences for genes PIK3C3, PIM3, and PTEN.

\begin{tabular}{lccc}
\hline Gene name & Forward primer sequence & Reverse primer sequence & TaqMan probe sequence \\
\hline PIK3C3 & GCCGATGATGAGGATTTGTTGATG & CCTTCTTGGTAGGTTCCAATCCATT & FAM-CCAGGCTCTCAAATAT \\
PIM3 & CGCTACCACCGCTACCA & CCCACACACCATATCGTAGAGAAG & FAM-ACGCCCAGCGACCAC \\
PTEN & GCACTGTTGTTTCACAAGATGATGT & ACCACAAACTGAGGATTGCAAGT & FAM-CCGCCACTGAACATT \\
\hline
\end{tabular}

TABLE 2: Average fold difference (FD) in the mRNA expression levels of PIK3C3, PIM3, and PTEN in patients with invasive cancer (stages I-IV) relative to patients with noninvasive cancer (stage 0 ).

\begin{tabular}{lcccccc}
\hline \multirow{2}{*}{ Cancer type } & \multicolumn{2}{c}{ PIK3C3 } & \multicolumn{2}{c}{ PIM3 } & \multicolumn{2}{c}{$P T E N$} \\
& FD & $P$ value & FD & $P$ value & FD & $P$ value \\
\hline Breast & 3.1 & 0.01 & 3.8 & 0.00 & 3.1 & 0.04 \\
Colon & 1.1 & 0.98 & 1.0 & 0.94 & 1.1 & 0.91 \\
Kidney & 0.6 & 0.07 & 0.9 & 0.41 & 1.0 & 0.52 \\
Liver & 0.8 & 0.38 & 0.8 & 0.21 & 0.7 & 0.09 \\
Lung & 1.3 & 0.77 & 1.1 & 0.83 & 1.6 & 0.19 \\
Ovary & 1.9 & 0.62 & 1.3 & 0.55 & 1.3 & 0.39 \\
Prostate & 0.7 & 0.35 & 1.3 & 0.61 & 0.8 & 0.21 \\
Thyroid & 0.5 & 0.03 & 0.8 & 0.26 & 1.3 & 0.23 \\
\hline
\end{tabular}

disease stage-averaged mRNA expressions for the genes was observed in colon, kidney, liver, lung, ovary, and prostate cancers. Neither of the disease stage-averaged mRNA expressions for PIM3 and PTEN was statistically significantly altered in thyroid cancer. These data suggested that disease stageaveraged mRNA expressions for genes PIK3C3, PIM3, and $P T E N$ could be used for breast cancer diagnosis but not for the other types of cancer. Since previous studies have shown upregulation of PIM3 or downregulation of PTEN in various types of cancer [33-36], the disease stage-averaged method may have neglected important information about the mRNA expressions of the three genes.

In order to get a better understanding on the expressions of PIK3C3, PIM3, and PTEN, we compared their mRNA expression levels at each disease stage (Figure 1). The mRNA expressions of all three genes varied significantly over the disease progression. In breast cancer, the mRNA expression levels of PIK3C3 and PIM3 were steadily increased during disease progression and elevated by 4.1- and 5.0fold, respectively, at stage IV. The mRNA expression level of PTEN was also augmented at each disease stage with a maximum of 3.6-fold increase at stage IIIC. In colon cancer, the mRNA expression of PIK3C3 decreased by about $40 \%$ at stage IIA and then gradually increased with a maximum of 50\% upregulation at stage IIIC. The mRNA expression of PIM3 was not altered over the disease progression; however, the mRNA expression of PTEN fluctuated with a significant 5 -fold decrease at stage III. In kidney cancer, the mRNA expression of PIK3C3 slowly decreased as the disease progressed, whereas the mRNA expressions of PIM3 and PTEN were both bell-shaped with a maximum of $40 \%$ and $50 \%$ increases, respectively, at stage II. As the disease approached stage IV, the mRNA expression levels of all three genes were downregulated by at least 2 -fold. In liver cancer, the change of the mRNA expression levels of the three genes was not significant except for stage IV. At stage IV, the expression level of PTEN was decreased by 2 -fold. In lung cancer, both PIK3C3 and PIM3 gave a bell-shaped response towards disease progression with a maximum of 2.4- and 2.5 -fold increases, respectively, at stage IIIA, whereas the mRNA expression of PTEN increased continuously through the disease progression and reached a 2.9 -fold elevation by stage IV. In ovary cancer, the expression of PIK3C3 also exhibited a bell-shaped response towards disease progression with a 4.5 -fold increase in stage IC and a 2 -fold decrease in stage IV. In prostate cancer, the mRNA expression levels of PIK3C3 and PTEN were slightly downregulated and the mRNA expression level of PIM3 was slightly upregulated for stages I to III. In thyroid cancer, the mRNA expressions of PIK3C3 and PIM3 decreased dramatically in stages I-II and subsequently increased as the disease progressed. The mRNA expression level of PTEN was elevated through all disease stages with a maximum of $60 \%$ increase at stage IV. From the above analysis, a better understanding about the involvement of genes PIK3C3, PIM3, and PTEN in cancer development and progression was achieved by comparing their mRNA expressions at each disease stage of different types of cancer. It should be noteworthy that mRNA upregulation of tumor suppressor gene PTEN warrants neither increased protein level for PTEN as increases in mRNA expression do not necessarily translate proportionally into protein expression nor PTEN in the wild-type as silencing of tumor suppressor genes through mutations is very common in cancers. Although we could determine PTEN expression at the protein level by Western blot and the identity of the PTEN protein (wild-type or mutant protein) by protein sequencing in our research laboratory, it would be costly, time-consuming, and even unnecessary to do that for each cancer patient during clinical laboratory tests when mRNA changes were employed for cancer diagnosis.

Finally, we examined whether the mRNA expressions of PIK3C3, PIM3, and PTEN were correlated during cancer progression. The mRNA expression levels of these three genes were trended using a third-degree polynomial function (Figure 1), and their pair-wise correlation coefficients for both stages 0 -IV and stages 0 -III were calculated (Table 3 ). The small sample size associated with each cancer type precluded a complete analysis; nonetheless, valuable information was obtained from our limited analysis. Based on disease stages $0-\mathrm{IV}$ and following the correlation classification defined by Taylor [37], high correlations $(0.68 \leq r \leq 1.0)$ were observed between PIK3C 3 and PIM3 in breast cancer, between PIK3C3 and PTEN in breast and ovary cancers, and between PIM3 and PTEN in breast, kidney, liver, and thyroid cancers. Moderate correlation $(0.34 \leq r \leq 0.67)$ was detected between 


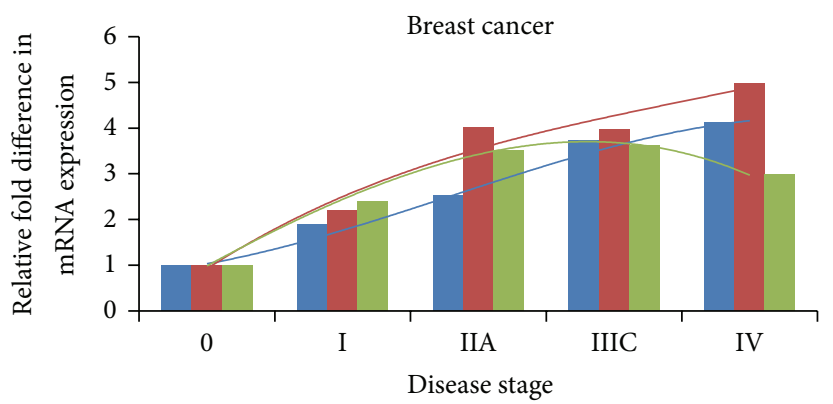

(a)

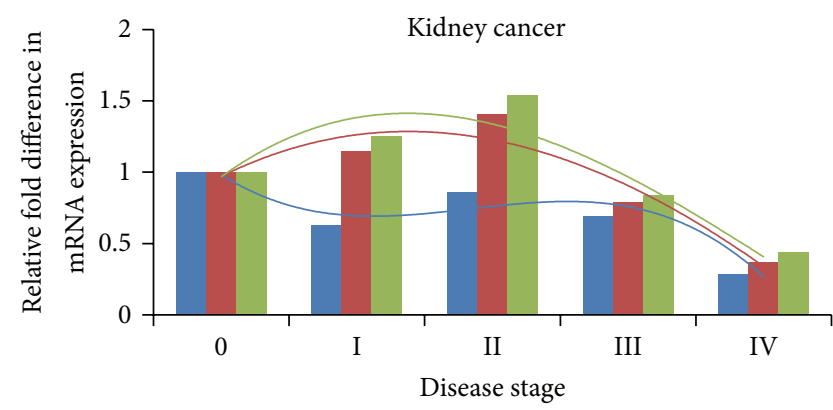

(c)

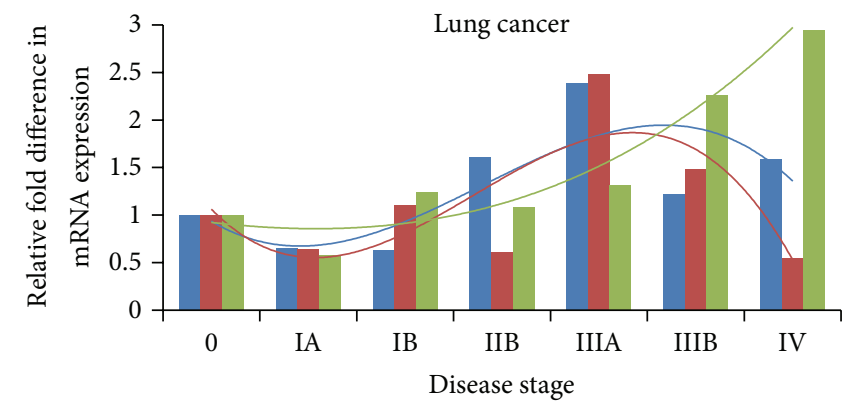

(e)

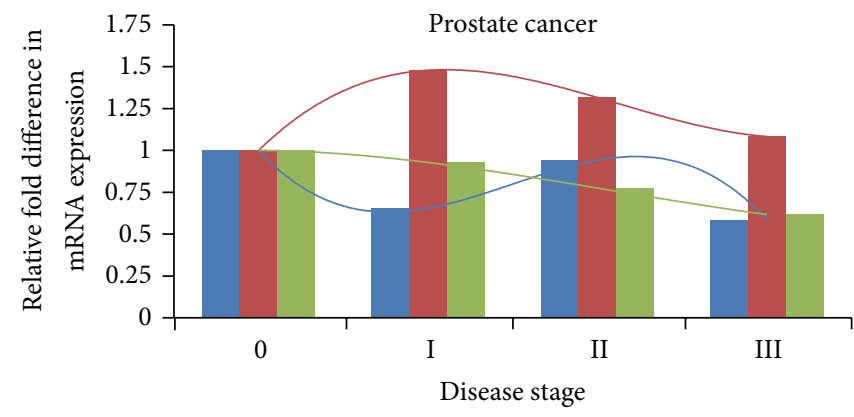

(g)

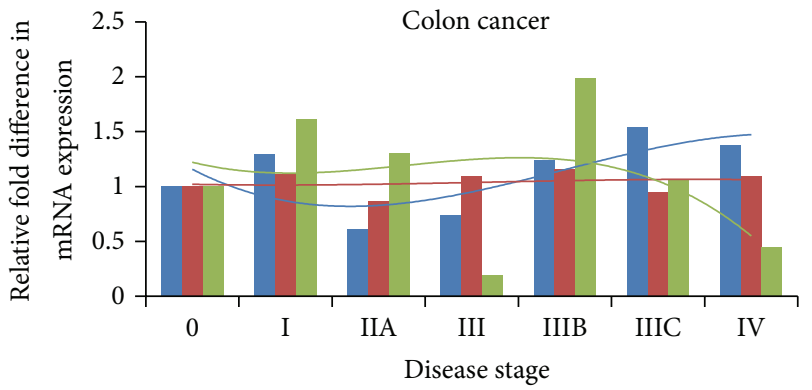

(b)

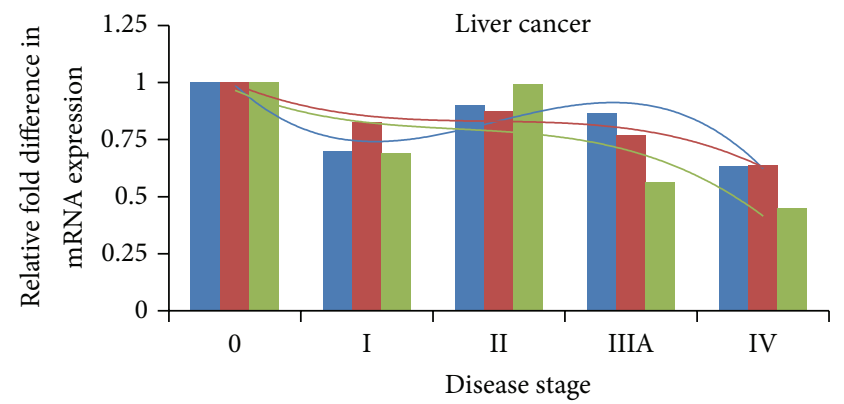

(d)

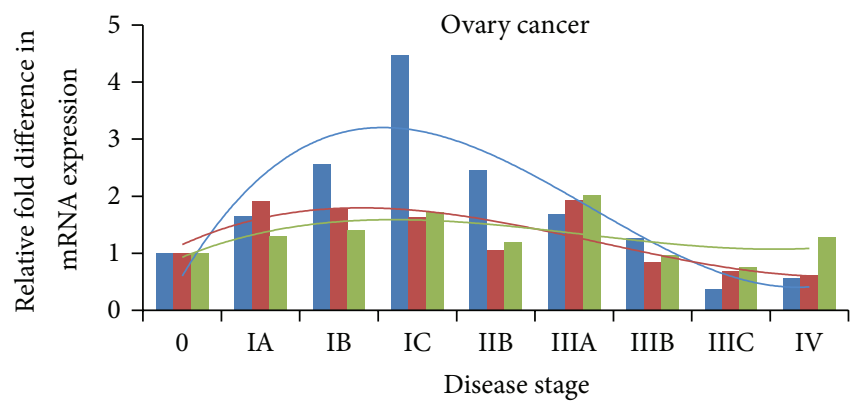

(f)

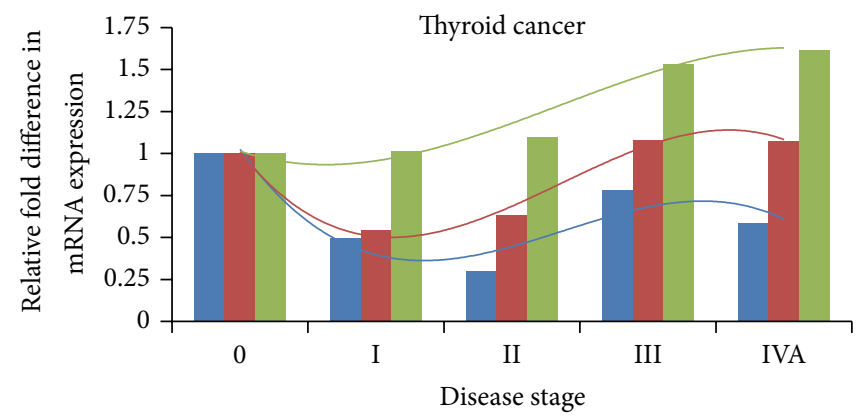

(h)

Figure 1: Relative fold difference in mRNA expressions of PIK3C3 (blue), PIM3 (coral), and PTEN (green) at different disease stages of breast (a), colon (b), kidney (c), liver (d), lung (e), ovary (f), prostate (g), and thyroid (h) cancers. The mRNA expression for each gene was trended using a third-degree polynomial function over the disease progression. Fold difference in mRNA expression at each disease stage was determined by comparison to the expression level in noninvasive cancer patients (stage 0 , expression level set as 1 ).

PIK3C3 and PIM3 in ovary cancer. Since RSR is much lower in patients with metastatic cancer, we compared the correlation coefficients for the mRNA expressions of the three genes between stages $0-$ IV and stages 0 -III (Table 3) in an attempt to find out whether these correlation coefficients could provide any hint of cancer metastasis. The change from statistically insignificant moderate correlation for stages 0 III to statistically significant high correlation for stages 0 -IV for the mRNA expressions of PIM3 and PTEN was observed in kidney and thyroid cancers, suggesting that the change in 
TABLE 3: Pair-wise comparisons of the mRNA expression levels of PIK3C3, PIM3, and PTEN during disease progression in eight cancer types with high $(0.68 \leq r \leq 1.00)$, moderate $(0.36 \leq r \leq 0.67)$, and low $(r \leq 0.35)$ correlations were shown as $(*),(* *)$, and $(* * *)$, respectively. Prostate cancer does not contain stage IV samples.

\begin{tabular}{|c|c|c|c|}
\hline \multirow{2}{*}{$\begin{array}{l}\text { Cancer } \\
\text { type }\end{array}$} & \multirow{2}{*}{ Gene pair } & \multicolumn{2}{|c|}{ Correlation coefficient $(r)$} \\
\hline & & Stages 0-IV & Stages 0-III \\
\hline \multirow{3}{*}{ Breast } & PIK3C3-PIM3 & $0.77(P<0.05)^{*}$ & $0.76(P<0.05)^{*}$ \\
\hline & PIK3C3-PTEN & $0.70(P<0.05)^{*}$ & $0.77(P<0.05)^{*}$ \\
\hline & PIM3-PTEN & $0.77(P<0.05)^{*}$ & $0.79(P<0.05)^{*}$ \\
\hline \multirow{3}{*}{ Colon } & PIK3C3-PIM3 & $-0.17(P>0.05)^{* * *}$ & $-0.18(P>0.05)^{* * *}$ \\
\hline & PIK3C3-PTEN & $0.35(P>0.05)^{* * *}$ & $0.41(P>0.05)^{* *}$ \\
\hline & PIM3-PTEN & $-0.22(P>0.05)^{* * *}$ & $-0.20(P>0.05)^{* * *}$ \\
\hline \multirow{3}{*}{ Kidney } & PIK3C3-PIM3 & $0.52(P>0.05)^{* *}$ & $-0.30(P>0.05)^{* * *}$ \\
\hline & PIK3C3-PTEN & $0.40(P>0.05)^{* *}$ & $-0.25(P>0.05)^{* * *}$ \\
\hline & PIM3-PTEN & $0.82(P<0.05)^{*}$ & $0.56(P>0.05)^{* *}$ \\
\hline \multirow{3}{*}{ Liver } & PIK3C3-PIM3 & $0.49(P>0.05)^{* *}$ & $0.54(P>0.05)^{* *}$ \\
\hline & PIK3C3-PTEN & $0.51(P>0.05)^{* *}$ & $0.51(P>0.05)^{* *}$ \\
\hline & PIM3-PTEN & $0.82(P<0.05)^{*}$ & $0.79(P<0.05)^{*}$ \\
\hline \multirow{3}{*}{ Lung } & PIK3C3-PIM3 & $0.41(P>0.05)^{* *}$ & $0.52(P>0.05)^{* *}$ \\
\hline & PIK3C3-PTEN & $0.44(P>0.05)^{* *}$ & $0.38(P>0.05)^{* *}$ \\
\hline & PIM3-PTEN & $0.21(P>0.05)^{* * *}$ & $0.50(P>0.05)^{* *}$ \\
\hline \multirow{3}{*}{ Ovary } & PIK3C3-PIM3 & $0.65(P<0.05)^{* *}$ & $0.61(P<0.05)^{* *}$ \\
\hline & PIK3C3-PTEN & $0.71(P<0.05)^{*}$ & $0.77(P<0.05)^{*}$ \\
\hline & PIM3-PTEN & $0.50(P>0.05)^{* *}$ & $0.60(P=0.05)^{* *}$ \\
\hline \multirow{3}{*}{ Prostate } & PIK3C3-PIM3 & & $0.29(P>0.05)^{* * *}$ \\
\hline & PIK3C3-PTEN & & $-0.23(P>0.05)^{* * *}$ \\
\hline & PIM3-PTEN & & $0.56(P>0.05)^{* *}$ \\
\hline \multirow{3}{*}{ Thyroid } & PIK3C3-PIM3 & $0.47(P>0.05)^{* *}$ & $0.29(P>0.05)^{* * *}$ \\
\hline & PIK3C3-PTEN & $0.11(P>0.05)^{* * *}$ & $-0.23(P>0.05)^{* * *}$ \\
\hline & PIM3-PTEN & $0.75(P<0.05)^{*}$ & $0.58(P>0.05)^{* *}$ \\
\hline
\end{tabular}

the correlation coefficient for the mRNA expressions of PIM3 and PTEN might be able to predict metastasis in kidney and thyroid cancers.

In summary, by using genes PIK3C3, PIM3, and PTEN as test samples, we showed that constructing biomolecular networks for normal and cancerous states and monitoring the correlations for the expressions of genes in the networks is a promising novel strategy in detecting tumorigenesis, cancer progression, and even cancer metastasis. Further studies involving confirmed biomolecular networks and a larger patient population certainly warrant investigations given the potential benefit for early cancer diagnosis and cancer patient survival. It is also important to address whether the biomolecular networks change during cancer progression in the future studies.

\section{Conflict of Interests}

The authors declare that there is no conflict of interests regarding the publication of this paper.

\section{Acknowledgments}

This work was supported in part by research grants from the Cancer Research Society, Canada, and the Canadian Breast Cancer Foundation.

\section{References}

[1] Canadian Cancer Society, Canadian Cancer Statistics 2013, Canadian Cancer Society, 2013.

[2] A. M. Ugnat, L. Xie, J. Morriss, R. Semenciw, and Y. Mao, "Survival of women with breast cancer in Ottawa, Canada: variation with age, stage, histology, grade and treatment," British Journal of Cancer, vol. 90, no. 6, pp. 1138-1143, 2004.

[3] R. A. Weinberg, The Biology of Cancer, Garland Science, New York, NY, USA, 2007.

[4] C. V. Dang, "MYC on the path to cancer," Cell, vol. 149, no. 1, pp. 22-35, 2012.

[5] M. Wade, Y.-C. Li, and G. M. Wahl, "MDM2, MDMX and p53 in oncogenesis and cancer therapy," Nature Reviews Cancer, vol. 13, no. 2, pp. 83-96, 2013.

[6] K. D. Cole, H.-J. He, and L. Wang, "Breast cancer biomarker measurements and standards," Proteomics: Clinical Applications, vol. 7, no. 1-2, pp. 17-29, 2013.

[7] V. M. Velonas, H. H. Woo, C. G. dos Remedios, and S. J. Assinder, "Current status of biomarkers for prostate cancer," International Journal of Molecular Sciences, vol. 14, no. 6, pp. 11034-11060, 2013.

[8] I. G. Yulug and B. Gur-Dedeoglu, "Functional genomics in translational cancer research: focus on breast cancer," Briefings in Functional Genomics and Proteomics, vol. 7, no. 1, pp. 1-7, 2008.

[9] F. Pandolfi, R. Cianci, D. Pagliari et al., “The immune response to tumors as a tool toward immunotherapy," Clinical and Developmental Immunology, vol. 2011, Article ID 894704, 12 pages, 2011.

[10] A. Stojanovic and A. Cerwenka, "Natural killer cells and solid tumors," Journal of Innate Immunity, vol. 3, no. 4, pp. 355-364, 2011.

[11] A. Carracedo and P. P. Pandolfi, "The PTEN-PI3K pathway: of feedbacks and cross-talks," Oncogene, vol. 27, no. 41, pp. 55275541, 2008.

[12] W.-S. Wu, J.-R. Wu, and C.-T. Hu, "Signal cross talks for sustained MAPK activation and cell migration: the potential role of reactive oxygen species," Cancer and Metastasis Reviews, vol. 27, no. 2, pp. 303-314, 2008.

[13] B. Zhu, X. Li, Y. Zhang et al., "Cross-talk of alpha tocopherolassociated protein and JNK controls the oxidative stressinduced apoptosis in prostate cancer cells," International Journal of Cancer, vol. 132, no. 10, pp. 2270-2282, 2013.

[14] J. S. Logue and D. K. Morrison, "Complexity in the signaling network: insights from the use of targeted inhibitors in cancer therapy," Genes \& Development, vol. 26, no. 7, pp. 641-650, 2012.

[15] V. Fodale, M. Pierobon, L. Liotta, and E. Petricoin, "Mechanism of cell adaptation: when and how do cancer cells develop chemoresistance?" The Cancer Journal, vol. 17, no. 2, pp. 89-95, 2011.

[16] F. V. Filipp, "Cancer metabolism meets systems biology: pyruvate kinase isoform PKM2 is a metabolic master regulator," Journal of Carcinogenesis, vol. 12, article 14, 2013. 
[17] T. M. K. Cheng, S. Gulati, R. Agius, and P. A. Bates, "Understanding cancer mechanisms through network dynamics," Briefings in Functional Genomics, vol. 11, no. 6, pp. 543-560, 2012.

[18] D. C. Y. Fung, A. Lo, L. Jankova et al., "Classification of cancer patients using pathway analysis and network clustering," in Network Biology, vol. 781 of Methods in Molecular Biology, pp. 311-336, Humana Press, New Jersey, NJ, USA, 2011.

[19] J. Chen, L. Sam, Y. Huang et al., "Protein interaction network underpins concordant prognosis among heterogeneous breast cancer signatures," Journal of Biomedical Informatics, vol. 43, no. 3, pp. 385-396, 2010.

[20] M. F. Islam, M. M. Hoque, R. S. Banik et al., "Comparative analysis of differential network modularity in tissue specific normal and cancer protein interaction networks," Journal of Clinical Bioinformatics, vol. 3, article 19, 2013.

[21] P. Workman and R. L. M. van Montfort, "Unveiling the secrets of the ancestral PI3 kinase Vps34," Cancer Cell, vol. 17, no. 5, pp. 421-423, 2010.

[22] Y. Takahashi, M. M. Yong, J. M. Serfass, T. Hori, and H. G. Wang, "Sh3glb1/Bif-1 and mitophagy: acquisition of apoptosis resistance during Myc-driven lymphomagenesis," Autophagy, vol. 9, no. 7, pp. 1107-1109, 2013.

[23] N. Mukaida, Y.-Y. Wang, and Y.-Y. Li, "Roles of Pim-3, a novel survival kinase, in tumorigenesis," Cancer Science, vol. 102, no. 8, pp. 1437-1442, 2011.

[24] M. Narlik-Grassow, C. Blanco-Aparicio, Y. Cecilia et al., "The essential role of PIM kinases in sarcoma growth and bone invasion," Carcinogenesis, vol. 33, no. 8, pp. 1479-1486, 2012.

[25] Y.-Y. Li, B. K. Popivanova, Y. Nagai, H. Ishikura, C. Fujii, and N. Mukaida, "Pim-3, a proto-oncogene with serine/threonine kinase activity, is aberrantly expressed in human pancreatic cancer and phosphorylates bad to block bad-mediated apoptosis in human pancreatic cancer cell lines," Cancer Research, vol. 66, no. 13, pp. 6741-6747, 2006.

[26] M. S. Song, L. Salmena, and P. P. Pandolfi, "The functions and regulation of the PTEN tumour suppressor," Nature Reviews Molecular Cell Biology, vol. 13, no. 5, pp. 283-296, 2012.

[27] Y. Kitagishi and S. Matsuda, "Redox regulation of tumor suppressor PTEN in cancer and aging: review," International Journal of Molecular Medicine, vol. 31, no. 3, pp. 511-515, 2013.

[28] A. J. Trimboli, C. Z. Cantemir-Stone, F. Li et al., "Pten in stromal fibroblasts suppresses mammary epithelial tumours," Nature, vol. 461, no. 7267, pp. 1084-1091, 2009.

[29] M. Y. Huang, H. C. Chen, I. P. Yang et al., “Tumorigenesis and tumor progression related gene expression profiles in colorectal cancer," Cancer Biomarkers, vol. 13, no. 4, pp. 269-279, 2013.

[30] I. Ratkaj, E. Stajduhar, S. Vucinic et al., "Integrated gene networks in breast cancer development," Functional \& Integrative Genomics, vol. 10, no. 1, pp. 11-19, 2010.

[31] M. Cieślik, S. A. Hoang, N. Baranova et al., "Epigenetic coordination of signaling pathways during the epithelialmesenchymal transition," Epigenetics \& Chromatin, vol. 6, article 28, 2013.

[32] Y. Yang, H. Li, S. Hou, B. Hu, J. Liu, and J. Wang, "Differences in gene expression profiles and carcinogenesis pathways involved in cisplatin resistance of four types of cancer," Oncology Reports, vol. 30, no. 2, pp. 596-614, 2013.

[33] H.-C. Zheng, K. Tsuneyama, H. Takahashi et al., "Aberrant Pim3 expression is involved in gastric adenoma-adenocarcinoma sequence and cancer progression," Journal of Cancer Research and Clinical Oncology, vol. 134, no. 4, pp. 481-488, 2008.
[34] B. K. Popivanova, Y.-Y. Li, H. Zheng et al., "Proto-oncogene, Pim-3 with serine/threonine kinase activity, is aberrantly expressed in human colon cancer cells and can prevent Badmediated apoptosis," Cancer Science, vol. 98, no. 3, pp. 321-328, 2007.

[35] W. Brenner, G. Färber, T. Herget, H.-A. Lehr, J. G. Hengstler, and J. W. Thüroff, "Loss of tumor suppressor protein PTEN during renal carcinogenesis," International Journal of Cancer, vol. 99, no. 1, pp. 53-57, 2002.

[36] N. Jones, F. Bonnet, S. Sfar et al., "Comprehensive analysis of PTEN status in breast carcinomas," International Journal of Cancer, vol. 133, no. 2, pp. 323-334, 2013.

[37] R. Taylor, "Interpretation of the correlation coefficient: a basic review," Journal of Diagnostic Medical Sonography, vol. 6, no. 1, pp. 35-39, 1990. 

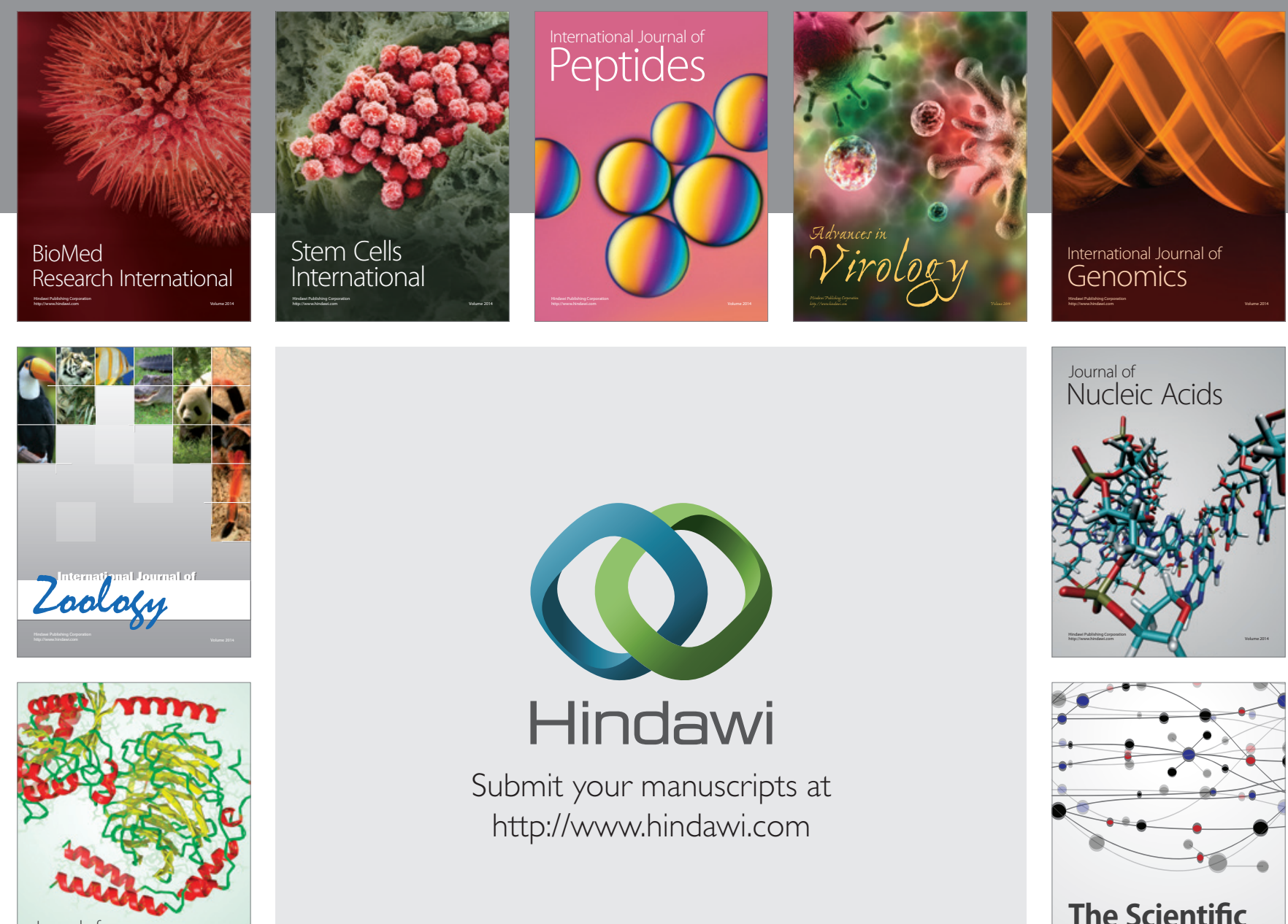

Submit your manuscripts at

http://www.hindawi.com

Journal of
Signal Transduction
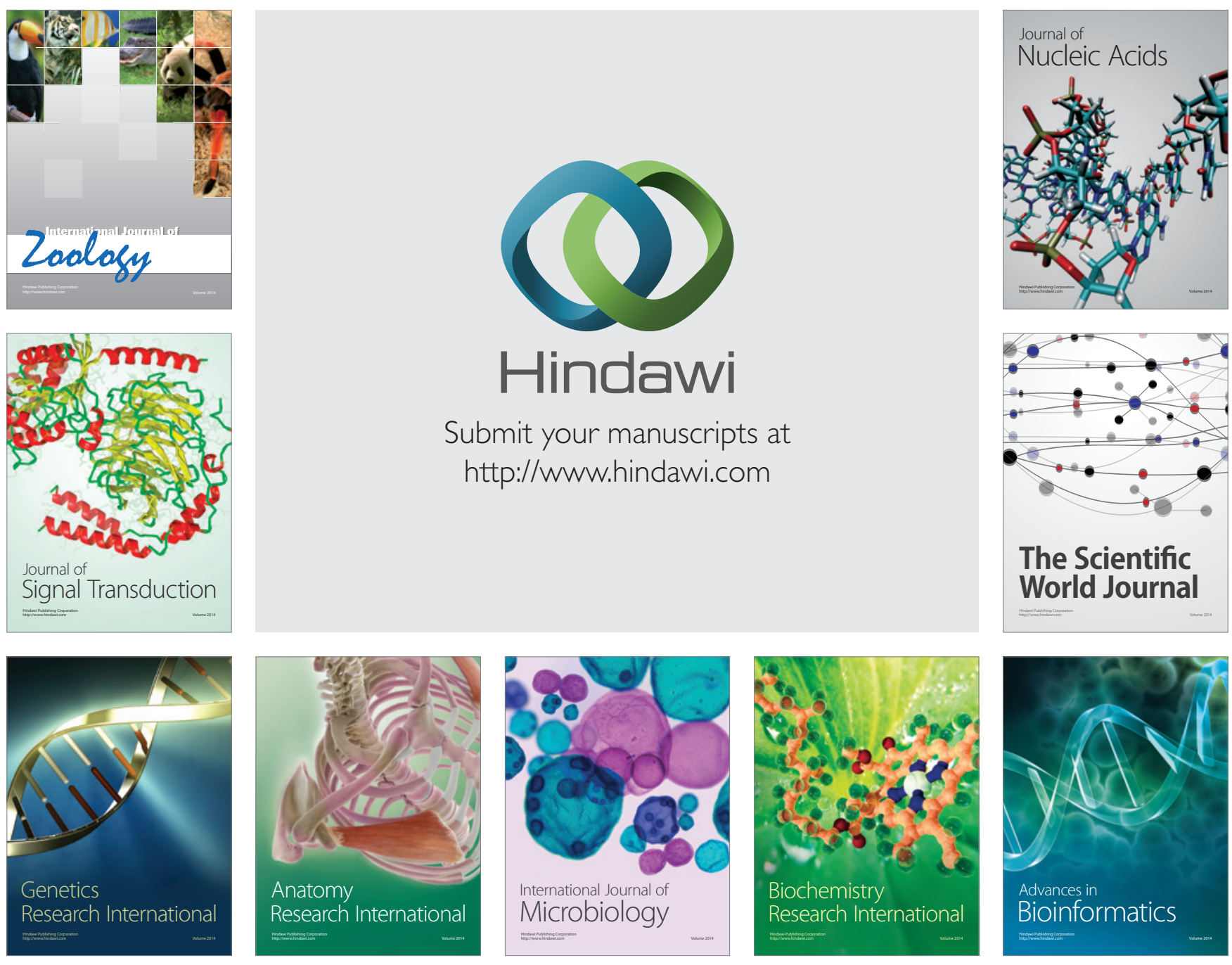

The Scientific World Journal
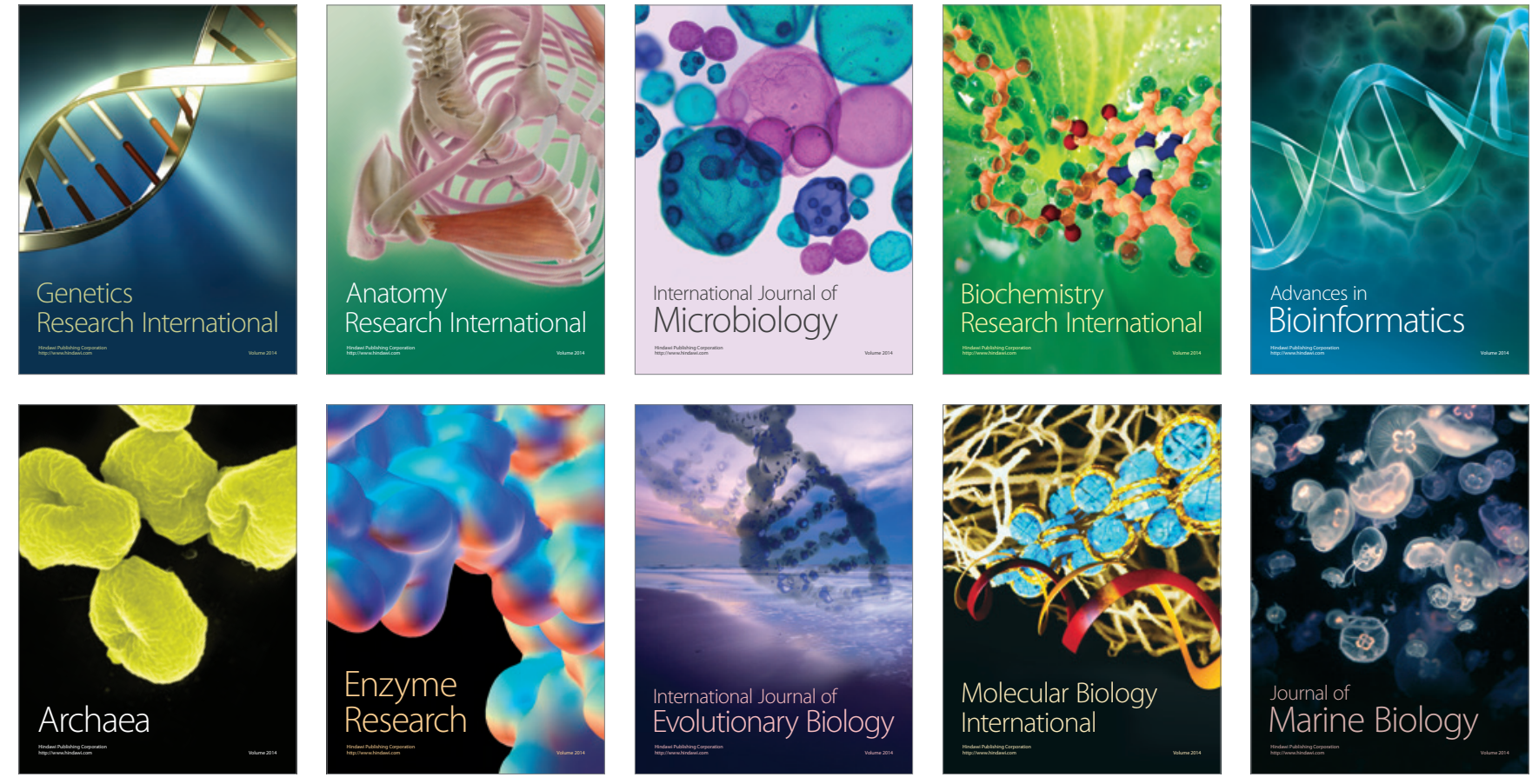